\title{
Los teoremas de la imposibilidad
}

\author{
Selim Gómez Ávila \\ ${ }^{a}$ Centro de Investigación en Matemáticas (CIMA) de la UAEH.
}

\begin{abstract}
Resumen
Una de las nociones más importantes de cualquier teoría física es la distinción entre leyes generales y condiciones particulares de las mismas. La física teórica tiene como misión encontrar las leyes fundamentales que describen nuestro universo, pero también explicar en la medida de lo posible esas condiciones particulares. Los teoremas de imposibilidad son un recurso valioso que provee una de las pocas guías existentes para la búsquedad de nueva física que permita profundizar nuestra comprensión de la naturaleza.
\end{abstract}

Palabras Clave: física teórica, teoría cuántica de campo, nueva física

\section{La labor de la física teórica}

La física está parada en la línea que separa lo necesario de lo contingente, lo meramente posible de lo absolutamente imprescindible, las leyes inquebrantables de los accidentes impredecibles. De todas las contribuciones de Newton quizá la más importante es la distinción entre las leyes naturales, condiciones generales que restringen todos los sucesos, y las condiciones iniciales, que eligen de entre las situaciones consistentes con las reglas generales aquellas que son en efecto realizadas. Como dijo Sir Hermann Bondi (Duncan and Ronald, 1985): «No podemos pretender, y ni siquiera imaginar, una teoría del Universo que nos diga por qué el cúmulo de Virgo se formó en una región y nuestro sistema local en otra cercana a la primera.» El árbol ordenado de la historia de nuestro universo crece a partir de una semilla azarosa.

Para avanzar, la física tiene que meticulosamente separar lo que puede ser de lo que tiene que ser, esto es, destilar del cúmulo de las observaciones y los experimentos aquellas regularidades que pueden codificarse en nuevas leyes de la naturaleza, e identificar el puñado de propiedades -la posición y la velocidad en un momento dado, o la temperatura y el campo magnético, por ejemplo- en términos de las cuáles resulta tal o cuál posibilidad. Pero no nos conformamos con eso: la labor de la física teórica es profundizar en nuestro entendimiento. La relatividad general y la mecánica cuántica, los dos triunfos emblemáticos de la física del siglo XX, fueron descubrimientos de nuevas regularidades universales que incluían a las anteriormente conocidas, y delimitaban el dominio en el cuál imperaban. Hay otra posibilidad de avance: que podamos explicar un hecho sobre el

\footnotetext{
*Autor en correspondencia.

Correo electrónico: selim_gomez@uaeh.edu.mx (Selim Gómez Ávila)
}

mundo en términos de una ley, elevando lo contingente al estatus de necesario. Pensemos en Kepler, intentando ajustar las órbitas de los planetas conocidos a los sólidos platónicos, esto es, tratando de encontrar una ley que hiciera obligatorio lo que hoy entendemos como un accidente.

Nuestras mejores teorías, que predicen los resultados de muchos experimentos con precisiones portentosas, están construidas a partir de muchos elementos que podemos colocar en una jerarquía de necesidades. Algunos son necesarios para la consistencia matemática de la teoría; otros parecen ser contingentes, ingredientes que tenemos que seleccionar para definir la teoría que nos interesa. Conocemos estos ingredientes a través de la observación continua del mundo que nos rodea a muchísimas escalas, desde lo ínfimo hasta lo inmenso; pero no los entendemos todavía.

Por ejemplo, sabemos que los objetos que nos rodean están hechos de átomos, y que éstos son sistemas compuestos por un puñado de diminutas partículas subatómicas. Si miro alrededor de mi oficina, y levanto un pisapapeles o una engrapadora, puedo entender sus propiedades -su masa, su temperatura, su carga eléctrica, etcétera - en términos del arreglo de esos minúsculos componentes. El electrón, el protón y el neutrón son suficientes para explicar mucho de lo que veo. Pero siempre es posible preguntarse, ¿por qué esos tres? ¿por qué no hay otras partículas estables de materia? (Resulta que están ahí, también, los ligerísimos neutrinos, que no forman parte de los objetos que nos rodean, pero sí están presentes en nuestro entorno, aunque son muy difíciles de detectar.)

La física nuclear y de partículas ha conseguido el logro asombroso de colisionar protones y núcleos atómicos a energías lo bastante altas para revelar que en su interior esconden otros componentes minúsculos de la materia, los quarks. Protones y neutrones resultan ser combinaciones de dos tipos de quarks, 
bautizados como quark arriba (up) y quark abajo (down). De modo que todo lo que podemos tocar está hecho de electrones y de estos dos quarks, además de que continuamente somos atravesados por neutrinos electrónicos. Esta corta lista de componentes fundamentales es un insumo de nuestra mejor teoría de partículas, el Modelo Estándar; no tenemos una razón fundamental que nos diga por qué esas cuatro y no otras. Dados estos ingredientes esenciales, más algunos otros (como una lista de sus interacciones) podemos usar una receta en la forma de las leyes de la física, para cocinar los resultados de muchísimos experimentos; pero la cantidad y naturaleza de los ingredientes es una condición inicial que permanece inexplicada.

Pero aún es peor: los experimentos de la física del siglo pasado (sobre todo, pero no sólo, los realizados en aceleradores de partículas) han mostrado que el electrón, el neutrino y los quarks tipo up y down no son toda la historia. Hay versiones más pesadas de cada una de estas partículas, a saber: dos copias del electrón, que llamamos el mu y el tau, con sus respectivos neutrinos, dos copias del quark up, que llamamos encanto (charm) y cima (top), y dos copias del quark down, que llamamos extraño (strange) y fondo (bottom). Como, salvo la masa, son casi idénticos, decimos que el Modelo Estándar tiene una estructura de familia: son los primos gordos. Nadie sabe hasta ahora por qué existe esta replicación, que resulta ser una de las preguntas abiertas más importantes de la física contemporánea.

El Modelo Estándar es la teoría que conjunta toda esta información sobre la composición elemental de la naturaleza. Si bien su formulación representa un enorme triunfo de la ciencia, pensamos que no es una teoría final; contiene un gran número de condiciones iniciales. Por ejemplo, tampoco sabemos por qué el espacio tiene tres dimensiones, o por qué las interacciones entre partículas son las electromagnéticas, nucleares débiles y fuertes y gravitacionales. Buscar física más allá del Modelo Estándar significa también explicar alguna de esas condiciones iniciales, pasarla de la columna de lo contingente a la de lo necesario. Esa es la misión de la física teórica de partículas.

Tabla 1: Contenido de materia del Modelo Estándar

\begin{tabular}{lrr}
\multicolumn{3}{c}{ Tabla 1: Contenido de materia del Modelo Estándar } \\
\hline Generación I & Generación II & Generación III \\
\hline Quark u $($ up $)$ & Quark c (charm) & Quark t (top) \\
Quark d (down) & Quark s (strange) & Quark b (bottom) \\
Electrón $e^{-}$ & Muón $\mu$ & Tau $\tau$ \\
Neutrino $v_{e}$ & Neutrino $v_{\mu}$ & Neutrino $v_{\tau}$ \\
\hline
\end{tabular}

\section{El paisaje de la metateoría}

Una perspectiva moderna de todo esto es decir que tenemos metateorías, como la Teoría de Campo, que son demasiado generales para describir al mundo: son teorías acerca de las teorías, marcos de trabajo que nos permiten descartar teorías ambiguas o inconsistentes, pero son insuficientes para fijar una teoría específica. Las reglas de estas metateorías son naturalmente abstractas, pero estudiándolas podemos ganar intuición sobre las razones detrás del mundo que observamos.
Por ejemplo, volvamos a la estructura de familias del Modelo Estándar. Idealmente, explicar esta estructura quiere decir formular una teoría donde la replicación de partículas sea una consecuencia y no un insumo que se reduzca a lo que conocemos, pero haga alguna predicción diferente, y nos provea de alguna ventaja conceptual, computacional o fenomenológica.

¿Cómo formular una nueva teoría? Los ingredientes esenciales para una teoría del tipo que nos interesa - lo que hemos llamado una teoría de campo- son tres: un espaciotiempo donde todo ocurre, un conjunto de interacciones fundamentales entre las partículas, y una lista de las partículas de materia que describe la teoría. La estructura de familias del Modelo Estándar es un problema difícil porque concierne al tercer ingrediente, que en gran medida es el menos entendido. Mucha de la física teórica de la segunda mitad del siglo XX consistió en la exploración sistemática de los primeros dos ingredientes.

¿Cómo podemos asegurar que nuestra teoría contiene al Modelo Estándar, es decir, que se reduzca en algún límite a lo que ya sabemos? Es bastante intuitivo que es necesario que el espaciotiempo, las interacciones y las partículas de materia estén contenidas de algún modo en la nueva teoría que estamos contruyendo. Esto puede ocurrir si los ingredientes del Modelo Estándar son subconjuntos de los nuevos ingredientes, o si pueden emerger de ellos, como ocurriría por ejemplo si descubrimos que electrones, neutrinos y quarks están hechos de unos componentes más diminutos todavía.

Resulta ser (y éste es una de las cosas que hemos aprendido de la metateoría) que no es posible escoger independientemente los tres ingredientes. Es decir, una teoría con un espaciotiempo arbitrario, un conjunto de interacciones arbitrarios, y una lista de partículas de materia arbitrarias, puede no ser consistente.

Por ejemplo, algunas de estas teorías son inestables: predicen un universo que instantaneamente desaparece en lo que Sidney Coleman llamo "la mayor catástrofe ecológica posible". Otras presentan anomalías de norma, violaciones cuánticas de leyes clásicas de conservación (como por ejemplo la de la carga eléctrica) producidas por ciertas combinaciones de partículas de materia. Éstas teorías no pueden hacer predicciones físicas, porque sus observables dependen de cantidades arbitrarias, imposibles de determinar. Otra manera de decir esto es que la presencia de la anomalía impide definir probabilidades que sumen consistentemente $100 \%$. Por último, algunas requieren una cantidad infinita de información para poder hacer una predicción, lo cuál las vuelve computacionalmente inútiles. Dice el folclor de los computólogos que en algunas de las primeras computadoras estaba pegado un letrero con el acrónimo GIGO, que en español sería "entra basura, sale basura". Este caso es similar: las teorías con insumos inconsistentes no sirven porque no hacen predicciones, están enfermas de ambigüedad.

\section{Los teoremas de imposibilidad}

Los teoremas de imposibilidad son resultados que restringen la manera en la que distintas ideas pueden combinarse; nos permiten identificar las teorías inconsistentes. Los teoremas de imposibilidad funcionan como guías para moverse en la metateoría. Si una teoría concreta es un lugar específico en el paisaje 
de las teorías, los teoremas de imposibilidad son la señalización que nos dice por dónde no podemos movernos, o al menos clarifican cuál es el precio a pagar por movernos en cierta dirección (Streater, 2007). O para hacer una metáfora culinaria, son una guía de maridaje que nos dice cuáles ingredientes podemos combinar con cuáles para no obtener una teoría indigesta. Por ejemplo, las leyes de la termodinámica pueden verse como un teorema de imposibilidad que prohíbe las máquinas de movimiento perpetuo.

Una de las predicciones más inquietantes de la mecánica cuántica temprana era la existencia de correlaciones entre sistemas que habían interactuado en el pasado y se entraban en un estado que llamamos entrelazado, donde el conjunto no podía reducirse a la suma de sus partes. Einstein, Podolsky y Rosen intentaron usar estas correlaciones para mostrar que la mecánica cuántica no podía ser una teoría completa, utilizando para ello dos suposiciones heredadas de una visión clásica del mundo. La primera era la localidad, que podemos entender como la ausencia de acciones instantáneas a distancia: los resultados de dos experimentos suficientemente separados deben ser independientes. La segunda era el realismo, la noción de que es posible asignarle valores definidos incluso a propiedades que no han sido medidas.

En los años heroicos del desarrollo de la mecánica cuántica, varios físicos prominentes buscaron construir una teoría que reprodujera las predicciones exitosas de la mecánica cuántica sin incluir algunas de sus propiedades menos intuitivas. Estos esfuerzos se vieron fuertemente impactados por el trabajo de John Bell, quién formulo un teorema de imposibilidad que muestra que la localidad y el realismo son inconsistentes con las predicciones cuánticas, de modo que ninguna teoría física de variables ocultas locales puede reproducir todas las predicciones de la mecánica cuántica. Las búsquedas de teorías que reproduzcan los triunfos de la cuántica se ven limitadas entonces a teorías que violen una de las dos suposiciones. Existe una tercera opción, el superdeterminismo, que no reconoce la existencia de elecciones, posibilidades o probabilidades genuinas en el universo, lo que lo hace incompatible con el falsacionismo y por tanto con la ciencia como actualmente la entendemos (Bell, 1988).

Durante el desarrollo de la física nuclear fuerte, al estudiar las partículas inestables que podían formarse con combinaciones de los quarks ligeros up, down y strange, Nambu, Han y otros notaron la existencia de estados que aparentemente chocaban con un teorema de imposibilidad. Este resultado, que llamamos el teorema de la conexión espín-estadística, implicaba que dos quarks no podían ocupar estados iguales, y sin embargo parecían haberse medido estados de tres quarks idénticos. Para resolver este problema, había dos posibilidades: o una de las suposiciones del teorema de imposibilidad era incorrecta (lo que también hubiera representado una revolución conceptual), o los quarks poseían una propiedad de la materia completamente nueva, que hacía a los estados diferentes. Esta nueva propiedad fue bautizada como el color cuántico; ese fue el primer paso para la creación de la cromodinámica cuántica, hoy considerada la teoría que describe las interacciones nucleares fuertes.

Un ejemplo más cercano a la física contemporanea es el teorema de imposibilidad de Malament y Hegerfeldt, que dice que una teoría cuántica y relativista con un estado mínimo de energía no contiene estados de partículas localizables; esto suele interpretarse como la necesidad de formular estas teorías en términos de campos y no de partículas. De nuevo, hay búsquedas de alternativas, y de nuevo, estas búsquedas son guíadas por el teorema de imposibilidad. Esta es la fortaleza de los teoremas de imposibilidad: es por esto que no debemos verlos como obstáculos, sino como señales en el camino.

\section{La ruta hacia una teoría final}

Un sueño de muchos físicos es que este proceso un día termine. Es decir, que exista una metateoría sin condiciones iniciales que produzca de manera unívoca el Modelo Estándar a bajas energías; ese es el sueño de una teoría final (Weinberg, 1994). Aunque no sabemos si este sueño se verá realizado un día, su búsqueda es uno de los motores de la física teórica contemporánea.

El teorema de Coleman-Mandula (Mandula, 2015), uno de los teoremas de imposibilidad más famosos, nos dice que no podemos mezclar las simetrías del espacio tiempo con las de las interacciones, siempre y cuando se cumplan las siguientes condiciones:

1. Se cumple la relatividad especial, al menos localmente

2. Se cumplen las reglas de la probabilidad: el paso de estados iniciales a estados finales es unitario.

3. Existe una partícula estable que es la más ligera.

Este teorema de imposibilidad restringe fuertemente las teorías que intentan extender el Modelo Estándar. En las últimas décadas se ha explorado sistemáticamente una clase de teorías, llamadas teorías de gran unificación, que son las extensiones que podemos construir respetando las suposiciones del teorema de Coleman-Mandula, y que prometen acercarnos a una explicación fundamental de algunas de las propiedades intrigantes del Modelo Estándar. En particular, si buscamos que quarks y leptones se unifiquen a altas energías, éstas teorías contienen genéricamente una predicción dramática, el decaimiento del protón, cuya observación sería una clara señal de que la idea de gran unificación va en la dirección correcta. Podemos evitar el decaimiento del protón solamente si decidimos conformarnos con una unificación parcial (los leptones y los quarks son objetos diferentes a todas las escalas) o sí introducimos mecanismos que lo prohiban y que a su vez tengamos que explicar; por ejemplo, realizando la gran unificación en una teoría con dimensiones extra (Fornal and Grinstein, 2017). Este es un programa de investigación de gran relevancia, que sin embargo no ha producido aún frutos experimentales.

Pero hay otros caminos prometedores. Existen posibles soluciones al problema de la estructura de familias que justo nacen de rechazar la primer o la última suposición del teorema de Coleman-Mandula. Una idea es que la dimensionalidad del espacio en el que vivimos sea distinta, y provenga de un proceso dinámico: esta es la idea de las dimensiones extra. La otra es que a muy altas energías la naturaleza sea descrita por una 
teoría que no tiene ninguna escala, lo que se llama una teoría con simetría conforme. Esto requiere, entre otras cosas, que todos los términos de masa desaparezcan. Al no tener ninguna escala de masa, no hay una partícula más ligera y por tanto no hay teorema de imposibilidad.

No debemos mirar los teoremas de imposibilidad como estériles paredes que bloquean nuestra ruta. Al contrario, nos informan sobre los problemas que encontraremos, y contienen en sus suposiciones los costos que debemos pagar por eludirlos.

\section{English Summary}

\section{No-go theorems.}

\begin{abstract}
One of the critical notions of a physical theory is the distinction between general laws and particular conditions. The mission of theoretical physics is the discovery of the fundamental laws that describe our universe, but also the deepest possible explanation of these particular conditions. No-go theorems are a valuable resource that provide one of the few guides available
\end{abstract}

in the search for new physics that can improve our understanding of Nature.

Keywords:

Theoretical physics quantum field theory new physics.

\section{Referencias}

Bell, J. S., 1988. Speakable and Unspeakable in Quantum Mechanics (Collected Papers on Quantum Philosophy). Cambridge University Press.

Duncan, Ronald, 1985. La enciclopedia de la ignorancia : todo lo que es posible conocer sobre lo desconocido (Spanish Edition). Fondo de Cultura Economica.

Fornal, B., Grinstein, B., 2017. SU(5) Unification without Proton Decay. Phys. Rev. Lett. 119 (24), 241801.

DOI: $10.1103 /$ PhysRevLett . 119.241801

Mandula, J. E., 2015. Coleman-Mandula theorem. Scholarpedia 10 (2), 7476, revision \#147717.

DOI: $10.4249 /$ scholarpedia. 7476

Streater, R., 2007. Lost Causes in and beyond Physics. Springer.

Weinberg, S., 1994. El Sueno de una Teoria Final: La Busqueda de las Leyes Fundamentales de las Naturaleza / Dreams of a Final Theory (Biblioteca de Bolsillo (Editorial Critica)) (Spanish Edition). Critica (Grijalbo Mondadori). 